\title{
Rezensionen
}

\section{Vier im Visier}

TEACCH

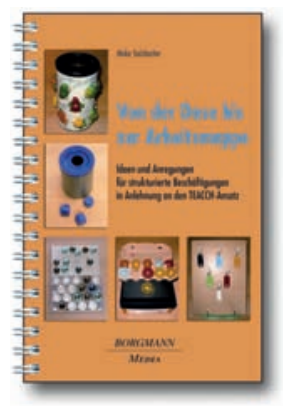

Lesen und direkt loslegen > Therapeuten und Eltern von Kindern mit Autismusspektrumstörungen (ASS) erhalten oft theoretische Informationen über verschiedenste Fördermöglichkeiten. Meist gestaltet es sich schwierig, alle Informationen in die Praxis umzusetzen. Heike Solzbacher arbeitet seit zwanzig Jahren mit Kindern mit ASS und hat Möglichkeiten entwickelt und niedergeschrieben, wie diese anhand des TEACCH-Ansatzes praktisch gefördert werden können. Das Buch ist keine weitere ausführliche theoretische Abhandlung, sondern erfreulich kurz, prägnant und anwenderbezogen. Es ermutigt den Leser mit zahlreichen Abbildungen direkt zum „Loslegen“. Wer mehr Interesse an einer fachlichen Vertiefung des TEACCH-Ansatzes hat, sollte entsprechende Fachliteratur hinzuziehen.

Im Schnelldurchlauf stellt Solzbacher allgemeine Strukturkonzepte und Umgebungs- bedingungen dar, um dann gründlich auf die vielen Übungsbeispiele einzugehen. Letztere sind für Klienten jeglichen Leistungsniveaus gedacht und von sehr einfachen praktischen bis hin zu anspruchsvolleren kognitiven Aufgaben gestaffelt. Einige Materialien sind nicht 1:1 im freien Handel erhältlich. Das ist von der Autorin bewusst gewünscht, da Material und Übungen individuell an den Klienten angepasst sein sollen. Einziger Kritikpunkt ist der nicht unerhebliche Zeitaufwand für die Herstellung einzelner Kisten, Dosen und Mappen.

Berücksichtigt man den Herstellungsaufwand, so ist das Buch besonders Therapeuten, Praxen und Einrichtungen zu empfehlen, da hier mehrere Kinder vom Material profitieren können. Aber auch Eltern erhalten wertvolle Tipps für Strukturierungsmöglichkeiten und Ideen zur Förderung ihres Kindes im Alltag. Bianka Kuhls, BSc (Occ Th), arbeitet im Therapiezentrum für autistische Kinder in Hannover

\section{H. Solzbacher:}

Von der Dose bis zur Arbeitsmappe.

Borgmann Media, Dortmund 2010.

$15,30 €$. 


\section{GEDÄCHTNISTRAINING}

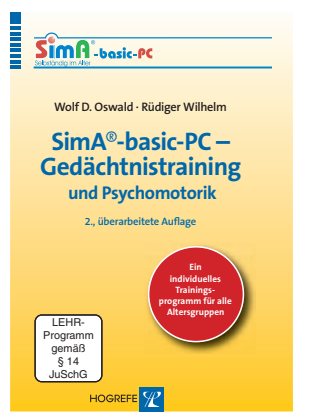

Gelungenes Programm > Nachlassende Gedächtnisleistungen gehören im Alter zu den Bereichen, die große Auswirkungen auf die alltägliche Lebensbewältigung haben. Ein regelmäßiges Gedächtnistraining in Verbindung mit einem regelmäßigen Training der Psychomotorik werten Wissenschaftler als beste Prävention, um zu verhindern, dass eine normale Altersdemenz fortschreitet. Bei bisher empfohlenen Trainingsaufgaben war es immer problematisch, dass die Aufgaben nach einmaliger Lösung bekannt waren und sich daher nicht zum regelmäßigen Training eigneten.

Das PC-Programm SimA-basic-PC überwindet diese Problematik. Die CD-ROM enthält 26 verschiedene Übungsaufgaben, die bei jedem Aufruf eine neue Aufgabenstellung bie- ten und mit denen der Klient deshalb über einen langen Zeitraum täglich üben kann. Am Ende jedes Übungsdurchgangs erhält der Nutzer eine Erfolgskontrolle, in der die Ergebnisse mit denen früherer Leistungen verglichen werden. So können Therapeuten diese $C D$ gut in den Therapiealltag integrieren. Zusätzlich umfasst das Programm Anleitungen zu 32 verschiedenen psychomotorischen Übungen in Form von kleinen Videos.

Ein Gedächtnistraining mit ständig variierenden Aufgaben für alle mit Computern vertrauten Senioren. Es empfiehlt sich für den Therapiealltag, aber auch für das Eigentraining zu Hause.

Verena Klinkner, Ergotherapeutin aus Mannheim

W. D. Oswald, R. Wilhelm:

SimA-basic-PC - Gedächtnistraining und Psychomotorik, CD-ROM.

2., überarb. Auflage,

Hogrefe Verlag, Göttingen 2010.

$39,95 €$.

\section{RATGEBER}

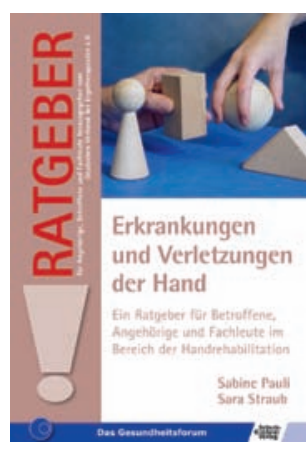

Nicht nur für Klienten > Sabine

Pauli betreut seit mehr als 20 Jahren Klienten mit Verletzungen und $\mathrm{Er}$ krankungen der Hand. Sie schrieb mehrere Fachbücher und Ratgeber. In diesem stellt die

Ergotherapeutin operative und konservative Behandlungsmöglichkeiten der Handtherapie vor und erläutert die Narbenbehandlung und Schienenversorgung sowie mögliche Begleiterscheinungen (Ödeme, Narben und Störungen der Sensibilität). Sie informiert über das Karpaltunnelsyndrom, rheumatische Erkrankungen und verschiedene Frakturen ebenso wie über Verbrennungen und Amputationen. Bei allen Krankheitsbildern erfährt der Leser neben möglichen Ursachen und Symptomen etwas über die Diagnostik und Behandlung der Erkrankung sowie die Möglichkeiten der Handtherapie. Schade, dass die Autorin nicht länger auf die Therapie bei Kindern eingeht. Hier wären mehr Informationen wichtig gewesen. Auch würde ich mir zusätzliche Abbildungen wünschen, da sie die Inhalte für Klienten verständlicher machen könnten.

Das Buch wendet sich an Klienten und ihre Angehörigen. Aber auch Ergo- und Physiotherapeuten erhalten etliche Informationen über verschiedene Krankheitsbilder, Verletzungen und allgemeine Behandlungsmöglichkeiten.

Katrin Steffen, Diplom-Ergotherapeutin (FH) aus Lingerhahn mit dem die Methode evaluiert werden kann.

Ein einfach umzusetzendes Programm, das man auch interdisziplinär nutzen kann. Es bietet Kindern einen klar strukturierten Ablauf sowie die Möglichkeit, sich spielerisch auf die schulischen Anforderungen einzustellen. Erfahrenen Ergotherapeuten werden diese Übungen weitgehend bekannt sein; Berufsanfängern und anderen Professionen können sie aber sicher viele Anregungen bieten.

Lina Wenninger, Bc (NL), Ergotherapeutin und freiberufliche Dozentin aus Essen erklärt. Oft sind Variationsmöglichkeiten beigebringen. Die Übungen greifen die Thematik werden im Verlauf anspruchsvoller. Teilweis sind sie etwas lieblos illustriert. Zu Beginn und am Ende des Programms müssen die Kinder

(n)
fahrten. Sie sind aufeinander aufgebaut und sodass die Kinder überschüssige Energie ablassen und ihre Grobmotorik schulen können. Anschließend machen sie Fingergymnastik und gehen zu grafomotorischen Aufgaben über.

Die Bewegungsspiele zur Fingergymnastik sind auf Foto-Karten abgebildet und rückseitig

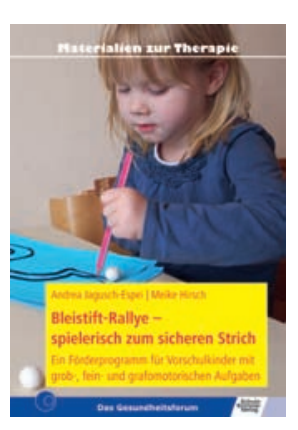

dergarten mit dieser Herausforderung. Die nen des Buchs, beide aus dem Berc die Grob-, Fein- und Grafomotorik vor schulkindern zu fördern. Es lässt sich schnell ten für die Eltern. Nach einem Begrüßungsritual startet die Stunde mit einem Bewegungsspiel,

\section{Mit Vollgas Rich-
tung Schule > Etli-} che Schulanfänger
kämpfen mit Stift, Für Schwungübungen ist im Schulalltag wenig Zeit. Deshalb beginnen Kin-

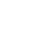

A.

\section{A. Jagusch-Espei, M. Hirsch: Bleistift-Rallye.}

Schulz-Kirchner Verlag, Idstein 2011. 38,99€.
S. Pauli, S. Straub:

Erkrankungen und Verletzungen der Hand. Schulz-Kirchner Verlag, Idstein 2011. $8,40 €$.

\section{LESEREMPFEHLUNG}

Wollen auch Sie Ihren Kolleginnen und Kollegen ein Buch empfehlen? Schicken Sie Ihren Vorschlag an: ergopraxis@thieme.de. Wir belohnen die besten Tipps mit einem Platz in ergopraxis und einem 20-Euro-Thieme-Buchgutschein! 